\title{
Dislocation transport and intermittency in the plasticity of crystalline solids
}

\author{
C. Fressengeas, ${ }^{1}$ A. J. Beaudoin, ${ }^{2}$ D. Entemeyer, ${ }^{1}$ T. Lebedkina,${ }^{3}$ M. Lebyodkin, ${ }^{1}$ and V. Taupin ${ }^{1}$ \\ ${ }^{1}$ Laboratoire de Physique et Mécanique des Matériaux, Université Paul Verlaine-Metz/CNRS, Ile du Saulcy, 57045 Metz Cedex, France \\ ${ }^{2}$ Department of Mechanical Sciences and Engineering, University of Illinois at Urbana-Champaign, 1206 West Green Street, \\ Urbana, Illinois 61801, USA \\ ${ }^{3}$ Institute of Solid State Physics, Russian Academy of Science, 142432 Chernogolovka, Russia \\ (Received 10 June 2008; revised manuscript received 10 September 2008; published 14 January 2009)
}

\begin{abstract}
When envisioned at the relevant length scale, plasticity of crystalline solids consists in the transport of dislocations through the lattice. In this paper, transport of dislocations is evidenced by experimental data gathered from high-resolution extensometry carried out on copper single crystals in tension. Spatiotemporal kinematic fields display spatial correlation through characteristic lines intermittently covered by plastic activity. Intermittency shows temporal correlation and power-law distribution of avalanche size. Interpretation of this phenomenon is proposed within the framework of a field dislocation theory attacking the combined problem of dislocation transport and long-range internal stress field development. Intermittency and transport properties show remarkable independence from sample size, aspect ratio, loading rate, and strain-rate sensitivity of the flow stress.
\end{abstract}

DOI: 10.1103/PhysRevB.79.014108

PACS number(s): 62.20.F-

\section{INTRODUCTION}

Transport is a convective process by which certain species, or variations in certain quantities, propagate in a medium. It is pervasive in many branches of physics. For example, transport serves as a cornerstone in the theory of fluid dynamics. Dislocations are linear defects connecting two parts of a crystalline solid sheared with respect to each other by a misfit referred to as the Burgers vector. They locally disrupt the ordering of atoms in the crystal and create displacement incompatibility, which results in lattice curvature and long-range elastic internal stress fields. Their motion through the lattice is responsible for the permanent plastic deformation of the crystal. When envisioned on length scales over which areal dislocation densities may be envisaged, dislocation motion is amenable to transport of these densities. The fundamental equation for dislocation transport has been known for half a century, ${ }^{1,2}$ mostly as a curiosity, and it is only recently that it has been effectively used for dislocation dynamics predictions. ${ }^{3}$ As yet however, the relevant length scale for the observation of dislocation transport remains elusive, although observation of strain waves ${ }^{4}$ could perhaps have given a clue. Here, we provide experimental evidence for dislocation transport at the appropriate scale of observation, i.e., in the micrometer to millimeter range. The results reveal an inherent connection between dislocation transport and the intermittency of plastic activity. These observations are substantiated through their interpretation within a field dislocation theory.

The intermittency of dislocation activity has been observed from time series for an even longer period of time. For example, it was described in $\mathrm{Zn}$ single crystals as early as $1932 .{ }^{5}$ However, the prevailing interpretation has been that in average over sufficiently large space and time scales, intermittent fluctuations add at random to a net smooth and homogeneous overall response. A fundamentally different picture emerged during the last few years when statistical analysis of these fluctuations became available, that of a scale-free phenomenon characterized by power-law distributions of avalanche size and correlations in space and time. Experimental evidence came from acoustic emission in ice single crystals, ${ }^{6,7}$ compression tests on fcc and bcc micropillars showing staircase-like stress-strain curves with powerlaw distribution of step size, ${ }^{8,9}$ and high-resolution extensometry experiments in $\mathrm{Cu}$ single crystals showing scaling behavior in the size of local strain-rate bursts. ${ }^{10}$ When oriented for multislip in tension, $\mathrm{Cu}$ single crystals represent the truly emblematic situation where material instability can be ruled out and homogeneous straining in a traditional sense expected. However, such crystals feature an inhomogeneous dislocation microstructure, which may well be at the origin of inhomogeneity through dislocation transport at a smaller scale. Hence they represent the perfect case for evidencing dislocation transport properties. In the present paper, this task is undertaken by tracking waviness in extensometry data. Based on optical methods, our experimental setup provides surface measurements, whereas crystal plasticity is a bulk phenomenon. Stored dislocation densities are known to be less close to the surface than in the bulk material, particularly in polycrystals. Hence, the surface is likely to be less hardened and more strained than the bulk. The differences in terms of strain can be significant in polycrystals but remain very small in single crystals, which ensure validity of the method.

Because they lack the spatial coupling due to internal stresses, conventional descriptions of plasticity cannot reproduce the scale-free behavior associated with intermittency of dislocation activity. Further, in a context of homogeneous hardening and in the absence of inertial forces, they fail to retrieve propagating fronts. By considering internal stresses due to dislocation-dislocation interactions, various modeling approaches such as two-dimensional (2D) (Ref. 7) and threedimensional (3D) (Ref. 11) discrete dislocation dynamics methods, phase field ${ }^{12}$ or statistical mechanics ${ }^{13}$ methods reproduce scale-free behavior. Both phase field and statisticalmechanics models use a quasistatic approach, either through energy minimization ${ }^{12}$ or presenting dislocation activity as 
"crackling noise"13 (see Ref. 14 for an introduction). Both fail to retrieve wave fronts of plastic activity as revealed by our experiments because they lack the hyperbolic character associated with the transport equation. Discrete dislocation dynamics simulations work at a smaller length scale: transport of dislocation densities is present but fully resolved into the motion of individual dislocations. Whether they can reproduce waves of plastic activity depends on their ability to cope with large enough systems in the future. In this paper, interpretation for the observed behavior is proposed within the framework of a field theory for dislocation dynamics dealing with the combined problem of dislocation transport and internal stress field development. ${ }^{15,16}$ In this theory, propagating fronts arise from consideration of transport. ${ }^{3,17}$ Two species of dislocation densities are considered depending on the scale of resolution. Resolved dislocation densities of net sign, referred to as "polar" or "geometrically necessary" dislocation densities, are regarded as a continuous manifestation of lattice incompatibility. They induce lattice curvature and long-range internal stress fields. Unresolved dislocation densities, also known as "statistical" dislocation densities, are the sole dislocation densities envisioned in conventional plasticity. Like polar dislocations, statistical dislocations have individual stress fields, but the latter statistically arranged so as to render a net zero overall field. Both species contribute to plasticity and their dynamics are coupled for gradients in plastic distortion rate may generate polar dislocations. In addition to evidencing transport and its intermittency, specific goals to forthcoming simulations are to investigate sample size and shape effects, as well as effects of loading rate and plastic slip behavior on their properties. The paper is organized as follows: in Sec. II, we present the highresolution extensometry experiments and their main results. The statistical analysis of these data is discussed in Sec. III. Section IV outlines the field dislocation theory used for their interpretation. Model simulations are shown and discussed in Sec. V. A summary and concluding remarks follow.

\section{HIGH-RESOLUTION EXTENSOMETRY}

In our experiments, $\mathrm{Cu}$ single crystals were oriented for multislip and strain hardening occurred immediately, without detectable stage I. The samples were loaded with constant crosshead velocity. They were clamped and no rotation of cross sections was observed before the onset of necking. The extensometry method is based on digital image correlation technique in one-dimensional setting. One side of the sample surface is painted in black, and strips of white paint normal to the longitudinal axis are superimposed, resulting in a network of black and white strips approximately $1 \mathrm{~mm}$ wide. The strips are bonded with the material underneath, and their displacement reflects perfectly the material displacement. A high-resolution charge-coupled device (CCD) camera with recording frequency $10^{3} \mathrm{~Hz}$ and pixel size of $1.3 \mu \mathrm{m}$ with the lens in use was mounted to capture the longitudinal displacement of points set at the intersection of the symmetry axis (to avoid any cross-section rotation effect) with the transitions between black and white strips. Fifteen to twenty such points were used, defining as many elementary exten- someters with gauge length about $1 \mathrm{~mm}$. From the onset of necking onward, strain localization occurs in a section of the sample, and the data are discarded as to the present purpose.

The displacements are derived to obtain the Lagrangian axial velocities then combined to produce axial strain rates. To avoid any bias due to possible differences in the sensitivity of the CCD camera to black-white vs white-black transitions, the latter are computed from displacement values at every two transitions. The driving strain rate $\dot{\epsilon}_{a}$ is kept constant throughout an experiment. Subtracting $\dot{\epsilon}_{a}$ from the strain-rate signal at a given material point leaves the variations shown in Fig. 1(b). Hence, the figure characterizes deviations from stationarity at this point. A positive excess in the figure results in less plastic activity at other locations along the sample, hence, in inhomogeneity of plastic straining. Despite smoothness of the loading curve [see Fig. 1(a)], the strain-rate variations [Fig. 1(b)] display jerks well above experimental noise level. At the relatively low stress values involved in this experiment, dislocation glide is controlled by local obstacles. It consists in successive fast runs of dislocation segments from one obstacle to the next one. The figure suggests that such jerkiness shows up at a somewhat larger scale. The probability density for the size of jerks shows power-law scaling [Fig. 1(c)], with scaling exponent $\tau \approx 2$ for both driving strain rates $\dot{\epsilon}_{a}=5 \times 10^{-4} \mathrm{~s}^{-1}$ and $\dot{\epsilon}_{a}=5$ $\times 10^{-3} \mathrm{~s}^{-1}$. This exponent is consistent with the scaling law reported for the associated acoustic emission. ${ }^{10}$ Such scaling is evidence for self-organization of the observed fluctuations, which is also suggested by Fig. 2. The figure features a space-time diagram for local fluctuations about the driving strain rate $\dot{\epsilon}_{a}=5 \times 10^{-4} \mathrm{~s}^{-1}$ during the elastoplastic transition. It shows spots of intense activity dotted along straight lines, suggesting wave propagation with maximum amplitude and average velocity amounting, respectively, to 2.5 $\times 10^{-3} \mathrm{~s}^{-1}$, and $10^{-2} \mathrm{~m} \mathrm{~s}^{-1}$. At larger strains, this wavy pattern is seen on shorter time and length scales due to decreasing dislocation free path in relation with multiplication of forest obstacles. Due to its jerky character, only statistical averages of the dislocation velocity are available in the literature. Strongly stress dependent, it is reported in the range of $10^{-6}-1 \mathrm{~m} \mathrm{~s}^{-1},{ }^{18}$ while the group velocity of dislocation ensembles is slower. The average wave velocity $V$ measured from the slopes of the characteristic lines in Fig. 2 (about $10^{-2} \mathrm{~m} \mathrm{~s}^{-1}$ ) is in that range. It is 5 orders of magnitude smaller than the velocity of elastic waves, but much larger than the material particles velocity, which suggests that the observed waves do reflect the underlying motion of dislocation ensembles. In this interpretation, the dotted pattern of spots along the characteristic lines is manifestation of the intermittency of dislocation motion. Analysis of intercorrelation between axial strain rates in two neighboring locations consistently shows strong anticorrelation over large periods of time. In addition, intercorrelation of a particular axial strain rate with all other strain rates shows alternatively correlation/anticorrelation [Fig. 1(d)]. This modulation is persistent when the gauge length for strain-rate evaluation is varied. In complement to Fig. 2, it is proof to the wavy structure of plastic activity, when the latter is envisioned at such length scales. 

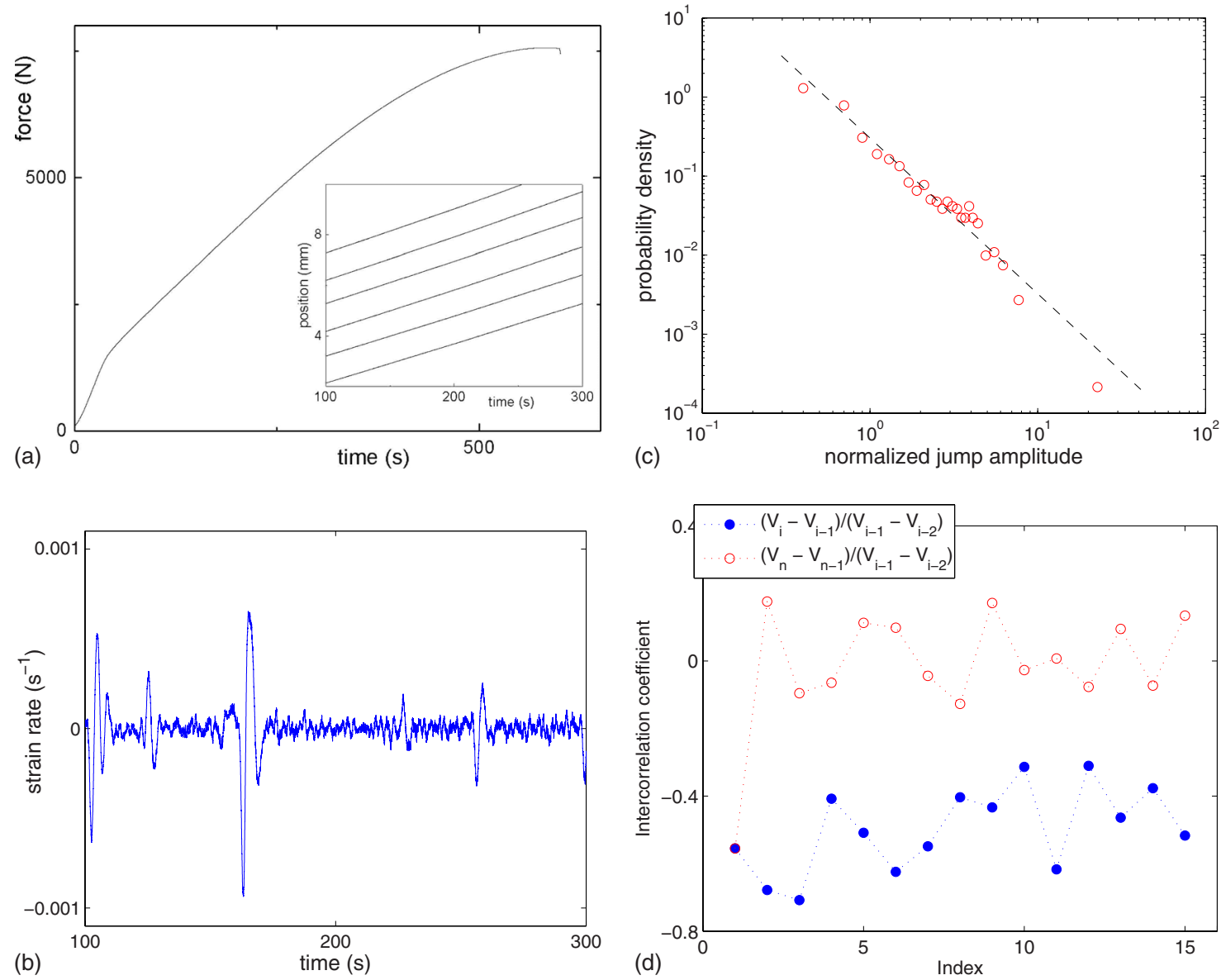

FIG. 1. (Color online) (a) Cu single crystal oriented for multislip under uniaxial tension (gauge length: $30 \mathrm{~mm}$, width: $5.5 \mathrm{~mm}$, thickness: $5.5 \mathrm{~mm}$, Schmid's factor: 0.3 , temperature: $20^{\circ} \mathrm{C}$, driving strain rate: $\dot{\epsilon}_{a}=5 \times 10^{-4} \mathrm{~s}^{-1}$ ), macroscopic force vs time (main graph) and displacement vs time in six locations distant by $1 \mathrm{~mm}$ (inset). (b) Variations in axial strain rate about the driving strain rate $\dot{\epsilon}_{a}$, as obtained from the lowest displacement curves in the stack in (a). Note that the size of the fluctuations can be larger than $\dot{\epsilon}_{a}$. (c) Probability density (normalized to bin size) for event size in time series shown in (b). The dashed line indicates the power-law trend with slope $\tau=2$. (d) Intercorrelation between strain rates derived from displacement signals at adjacent locations $\left(x_{i}, x_{i+1}\right)$ and $\left(x_{i+1}, x_{i+2} ; i=1,13\right)$ (blue dots); intercorrelation between strain rates derived from displacement signals at distant locations $\left(x_{1}, x_{2}\right)$ and $\left(x_{j}, x_{j+1} ; j=2,14\right)$ (red open circles). Same data except sample thickness: $2.3 \mathrm{~mm}$ and driving strain rate: $5 \times 10^{-3} \mathrm{~s}^{-1}$.

\section{STATISTICAL ANALYSIS}

Scaling regimes with exponent $\tau \approx 2$ in event size distribution have been reported using several techniques in various metallic and ice single crystals. ${ }^{6,7,10}$ Smaller exponents, albeit consistent with this value as shown below, were found in the distribution of integrated event size measures ${ }^{8}$ and retrieved using several modeling approaches. ${ }^{7,11-13}$ Hence, conjecturing universality in the sense of uniqueness of the scaling exponent for the distribution of event size is an appealing idea. However, universality claims need to account for several compounding factors, such as overlapping of avalanches due to finite driving rates (see, for example, Ref. 19) or variants in the involved statistics. We deal with the former in Sec. V and with the latter in the following by presenting our statistical approach as well as an overview of other methods. Usually the analyzed data $y(t)$, with maximum amplitude $Y$, is either the amplitude of bursts in material velocity $v(t)$ or in plastic strain rate $\dot{\boldsymbol{\epsilon}}(t)$ (as in the present experiments), or integral measures $S$ over a time interval $\tau: S$ $=\int_{t_{0}}^{t_{0}+\tau} y(t) d t$, such as displacement $u(t)$, strain $\epsilon(t)$, or stress $\sigma(t)$. The statistics of dislocation avalanches are then characterized by probability densities $p(Y)$ or $p(S)$. Other measures

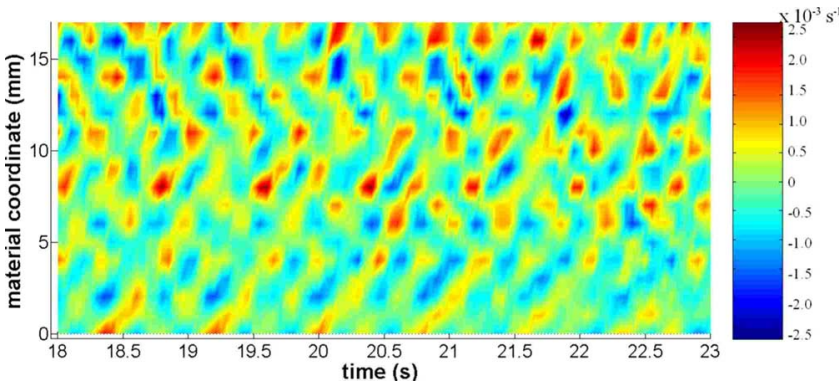

FIG. 2. (Color online) Longitudinal fluctuations about the imposed strain rate in a space-time diagram during the elastoplastic transition. Dotted characteristic lines run from the left and right of the gauge length, reflecting intermittency and transport. The imposed strain rate is $\dot{\epsilon}_{a}=5 \times 10^{-4} \mathrm{~s}^{-1}$. Fluctuations can be as high as $2.5 \times 10^{-3} \mathrm{~s}^{-1}$. 
have been used, such as the cumulative probability of events, $p(Y>L)$, in case the statistics are too poor, or the probability density $p(E)$ for energy release $E$ by acoustic emission due to dislocation avalanches. ${ }^{7}$ The latter is estimated either by integrating $\dot{\epsilon}^{2}$ or by using the squared maximum peak strainrate amplitude $Y^{2}$. Of course, the integrated measures depend on the burst shape, which may be loosely defined in the presence of noise, and several options can be considered. Clearly the difference in nature of all these quantities may imply differences in their respective distributions.

Due to noise (experimental or numerical), identification of slip events in time series may be ambiguous and, as a result, various definitions of event size have been employed. The most common approach, used in particular in acoustic emission measurements, consists of setting a threshold and defining an event as the continuing part of the signal whose magnitude is greater than the threshold. ${ }^{10}$ Then the event size may be defined either as the integral of the signal or as the peak amplitude of the event over that time spell. Another choice is to look for all local peaks, ignore those peaks whose value is too close to the surrounding level according to some chosen threshold, and then define events according to the selected peaks, whose amplitude is labeled $Y$. It was found in our data that both definitions of event size based on peak amplitude result in power-law distributions of probability density, $p(Y) \sim Y^{-\alpha}$, with $\alpha \approx 2$, similar to those displayed in Figs. 1 and 6. Power-law distributions were also obtained when event size was defined from integrated signals $p(S)$ $\sim S^{-\alpha_{S}}$ but, instead of $\alpha \approx 2$, the exponents were found in the range $1.2<\alpha_{s}<2$, depending on experimental conditions.

Assume accordingly that the probability density $p(Y)$ in the peak amplitude $Y$ follows a power law $p(Y) \sim Y^{-\alpha}$, and let $Z$ be a single-valued function of $Y$. We are interested in (i) the cumulative probability of events whose size is larger than $L: p(Y>L)$ and (ii) the relationship between the respective probability densities $p(Y)$ and $p(Z)$. $Z$ stands for either the integral measure $S$ or the acoustic emission energy $E$ mentioned above. In the former case (i) the cumulative probability of events also leads to scaling behavior, but since

$$
p(Y>L)=\int_{L}^{\infty} p(Y) d Y \sim \int_{L}^{\infty} Y^{-\alpha} d Y \sim Y^{-(\alpha-1)},
$$

the scaling exponent is $(\alpha-1)$. In the latter case (ii), $p(Y) d Y=p(Z) d Z=p(Z) d Z / d Y d Y$ and $p(Z)$ can clearly be expressed as

$$
p(Z)=\frac{p[Y(Z)]}{d Z / d Y} .
$$

Therefore, due to the denominator, $p(Z)$ depends on the burst shape. Several assumptions can be made to model avalanche decay.

(a) For the sake of simplicity, a triangular burst shape with linear decay is sometimes hypothesized. In this case, $\tau \sim Y$, $S=\tau Y \sim Y^{2}$, and Eq. (2) leads to $p(S) \sim S^{-\alpha_{S}}$ with $\alpha_{S}=(\alpha$ $+1) / 2$. Although a different scaling exponent is found, the scaling in $S$ is of course compatible with the scaling in $Y: p(Y) \sim Y^{-\alpha}$. (b) Alternatively, assume exponential avalanche decay: $y(t)=Y \exp \left[-\left(t-t_{0}\right) / \tau\right]$, as observed in acoustic emission data. ${ }^{10}$ Integration over time from the initial instant $t_{0}$ yields $S=\tau Y \sim Y$. Hence, according to Eq. (2), $S$ obeys the same power law, $p(S) \sim S^{-\alpha}$. This result is also approximately valid in the presence of noise. In this case, the burst duration can be defined as the time $\tau_{n}$ beyond which the signal falls below noise level, $Y_{n}$, and it is given by relation $\tau_{n}=\tau \ln \left(Y / Y_{n}\right)$. Integration of the signal over this period of time yields $S$ $=\tau\left(Y-Y_{n}\right)$, and therefore $d S / d Y$ does not depend on $Y$. Of course, $Y=S / \tau+Y_{n}$. It involves a correction from the noise level, which becomes small if $S$ is large enough. Hence, the power-law relation $p(S) \sim S^{-\alpha}$ is satisfied for large events, although deviation due to noise at small events may lead to underestimating $\alpha$. Therefore, using this scaling relation to characterize acoustic emission data in comparison with velocity data ${ }^{10}$ is fully consistent. (c) A third simple option is power-law avalanche decay: $y(t)=Y\left(t / t_{0}\right)^{-\beta}$. When the decay is fast $(\beta>1)$, integration leads to the same result as in case (b) for exponential decay: the $p(Y)$ and $p(S)$ distributions are obeying the same power-law. This conclusion is exact in the absence of noise and approximate for noisy signals. Thus both cases considered above (exponential and fast power-law decay) lead to identity of the scaling exponents $\alpha$ and $\alpha_{s}$. Different scaling behavior is now obtained in case of slow power-law decay: $\beta<1$. Modeling the signal in this way makes sense only in the presence of noise since the latter removes divergence associated with integration. The approximate solution $\left(Y_{n} / Y\right.$ must be small enough) then leads to $S \sim Y^{1 / \beta}$ and, according to Eq. (2), $p(S) \sim S^{-\alpha_{s}}$ with $\alpha_{s}$ $=1+\beta(\alpha-1)$. The discrepancy in the scaling exponents can be checked effectively as $\beta$ can be obtained from the measurement of the burst duration $\tau_{n}$ through the relations: $\tau_{n}$ $=t_{0}\left[\left(Y / Y_{n}\right)^{1 / \beta}-1\right] \cong t_{0}\left(Y / Y_{n}\right)^{1 / \beta}$, although estimating $\tau_{n}$ may prove a difficult task. Such differences were indeed found in our data: the scaling exponent $\alpha \approx 2$ shown in Fig. 2(c) corresponds to $\alpha_{s} \approx 1.3$ when an integrated measure of strainrate bursts is used. The exponent for burst decay is estimated as $\beta \approx 0.25$. Hence the relation $\alpha_{s}=1+\beta(\alpha-1)$ is approximately satisfied. The specific case $\beta=1$, which corresponds to Omori-type decay, ${ }^{20}$ leads to $0 / 0$ uncertainty. We note however that $\alpha_{s}$ tends to $\alpha$ when $\beta$ tends to 1 from below, while $\alpha_{s}=\alpha$ when $\beta>1$, as shown above.

Acoustic emission energy can be estimated as (i) $E \sim Y^{2}$ (Ref. 7) or (ii) as $E \sim \int y^{2}(t) d t$, where $y(t)$ stands for the strain-rate time series. In the former case (i), it can be easily shown from the above that $p(E) \sim E^{-\alpha_{E}}$ with $\alpha_{E}=(\alpha+1) / 2$ if $p(Y) \sim Y^{-\alpha}$. In the latter case (ii), the conclusion depends on the burst decay assumption. If burst decay is modeled as linear or exponential, the result $\alpha_{E}=(\alpha+1) / 2$ holds. If power law is assumed instead, a discussion similar to the above one shows that this result holds when the decay exponent $\beta$ is large enough $(\beta>1)$, but it gives way to the relation $\alpha_{E}=1$ $+\beta(\alpha-1)$ when burst decay is slow $(\beta<1)$.

According to the above analysis, a robust scaling exponent $\alpha \approx 2$ is found in the present experimental data for peaksize distributions, whatever choice of peak-size definition is made. Instead, the scaling exponent $\alpha_{S}$ for integrated measures displayed scattering. Such scatter does not contradict peak-size scaling as can be explained from various modeling 
assumptions for avalanche decay. However, peak-size analysis seems to be more reliable than integral-size analysis. It supports the notion of a universal power-law exponent, independent of specific microscopic structure or mechanical behavior.

\section{FIELD DISLOCATION MODEL}

We now provide interpretation for the intermittency of plasticity and substantiate the identification of surface waves with dislocation transport phenomena by the description of propagating fronts of plastic activity using a field dislocation model. The model uses the continuum description of dislocations based upon Nye's dislocation density tensor $\boldsymbol{\alpha} \cdot{ }^{21} \mathrm{Op}$ erating on the normal $\mathbf{n}$ to a unit surface $S, \boldsymbol{\alpha}$ provides the net Burgers vector $\mathbf{b}=\boldsymbol{\alpha} \cdot \mathbf{n}$ of all dislocations lines threading $S$, i.e., the incompatibility in plastic displacement found along the Burgers circuit $C$ surrounding $S$. When surface $S$ is so small that it is threaded by a single dislocation with Burgers vector $\mathbf{b}$ and line vector $\mathbf{t}, \boldsymbol{\alpha}=\mathbf{b} \otimes \mathbf{t}$ and the involved dislocation is labeled as a "polar dislocation." When the size of $S$, i.e., the resolution length scale, is increased to the point where $S$ is threaded by a large number of dislocations and $\mathbf{b}$ may become zero if all individual Burgers vectors compensate statistically. Then $\boldsymbol{\alpha}$ is zero, the dislocations are unresolved and deemed "statistical." In intermediate cases, the net Burgers vector $\mathbf{b}$ is nonzero, but part of the dislocations threading $S$ may remain unresolved. The subscripts in the density components $\alpha_{i j}$ then indicate the net Burgers vector and line vector directions of polar dislocations, whereas the remaining statistical dislocations are not accounted for. Due to lattice incompatibility, the plastic distortion tensor $\mathbf{U}_{\mathbf{p}}$ is not a gradient. It has compatible and incompatible parts, $\mathbf{U}_{\mathbf{P}}^{\|}$ and $\mathbf{U}_{\mathbf{P}}^{\perp}$, respectively, such that $\mathbf{U}_{\mathbf{p}}=\mathbf{U}_{\mathbf{p}}^{\|}+\mathbf{U}_{\mathbf{p}}^{\perp} \cdot{ }^{3}$ The incompatible part results from the distribution $\boldsymbol{\alpha}$ and is solution to the incompatibility equation,

$$
\operatorname{curl} \mathbf{U}_{\mathbf{p}}^{\perp}=-\boldsymbol{\alpha}
$$

written here at small strains, like all following equations. The elastic distortion tensor $\mathbf{U}_{\mathbf{e}}$ has an incompatible part $\mathbf{U}_{\mathbf{e}}^{\perp}$ opposed to $\mathbf{U}_{\mathbf{p}}^{\perp}$

$$
\mathbf{U}_{\mathbf{e}}^{\perp}+\mathbf{U}_{\mathbf{p}}^{\perp}=0
$$

so that the compatibility of the displacement gradient $\mathbf{U}$ $=\operatorname{grad} \mathbf{u}$ is ensured. The latter is written as

$$
\operatorname{grad} \mathbf{u}=\mathbf{U}_{\mathbf{e}}^{\|}+\mathbf{U}_{\mathbf{p}}^{\|},
$$

where $\mathbf{U}_{\mathbf{e}}^{\|}$is the compatible part of the elastic distortion tensor. Both $\mathbf{U}_{\mathbf{e}}^{\|}$and $\mathbf{U}_{\mathbf{p}}^{\|}$are gradient tensors, which implies $\operatorname{curl} \mathbf{U}_{\mathbf{e}}^{\|}=$curl $\mathbf{U}_{\mathbf{p}}^{\|}=0$. Hence Eq. (3) may be replaced with ${ }^{1}$

$$
\operatorname{curl} \mathbf{U}_{\mathbf{p}}=-\boldsymbol{\alpha}
$$

or, as well, with

$$
\operatorname{curl} \mathbf{U}_{\mathbf{e}}=\boldsymbol{\alpha} .
$$

Specifically solving Eq. (3) for $\mathbf{U}_{\mathbf{p}}^{\perp}$, without the gradient contribution present in Eq. (6), requires additionally

$$
\operatorname{div} \mathbf{U}_{\mathbf{p}}^{\perp}=0
$$

with boundary condition $\mathbf{U}_{\mathbf{p}}^{\perp} \cdot \mathbf{n}=0$ (here $\mathbf{n}$ is the outward normal to the sample external boundary). These conditions guarantee uniqueness of $\mathbf{U}_{\mathbf{p}}^{\perp}$. $^{3}$

When all dislocations are resolved, the plastic distortion rate $\dot{\mathbf{U}}_{\mathbf{p}}$ results from the motion of polar dislocations $\boldsymbol{\alpha}$ with velocity $\mathbf{V}$ (throughout the paper, a superposed dot indicates a time derivative). When averaging in space, statistical mobile dislocations are likely to be present at medium resolution scale. These dislocations contribute to the plastic distortion rate through the (unresolved) conventional plastic velocity gradient $\mathbf{L}_{\mathbf{p}}$. Therefore the total plastic distortion rate is, at this scale,, 15

$$
\dot{\mathbf{U}}_{\mathbf{p}}=\boldsymbol{\alpha} \times \mathbf{V}+\mathbf{L}_{\mathbf{p}}
$$

with dislocation velocity $\mathbf{V}$ now averaged over $S$. The incompatible part of $\dot{\mathbf{U}}_{\mathbf{p}}$ feeds the increment of polar dislocations through the transport equation for dislocation densities ${ }^{2}$

$$
\dot{\boldsymbol{\alpha}}=-\operatorname{curl} \dot{\mathbf{U}}_{\mathbf{p}} .
$$

This equation provides for the evolution and transport of polar dislocation densities. Through the curl of the total plastic distortion rate tensor $\dot{\mathbf{U}}_{\mathbf{p}}$, it couples the polar and statistical dislocation densities for the nucleation of polar dislocations. The stress tensor $\mathbf{T}$ is obtained from the tensor of elastic constants $\mathbf{C}_{\mathbf{e}}$ as

$$
\mathbf{T}=\mathbf{C}_{\mathbf{e}}:\left\{\mathbf{U}_{\mathbf{e}}\right\}=\mathbf{C}_{\mathbf{e}}:\left\{\mathbf{U}_{\mathbf{e}}^{\|}+\mathbf{U}_{\mathbf{e}}^{\perp}\right\}=\mathbf{C}_{\mathbf{e}}:\left\{\operatorname{grad} \mathbf{u}-\mathbf{U}_{\mathbf{p}}^{\|}-\mathbf{U}_{\mathbf{p}}^{\perp}\right\},
$$

where $\{\mathbf{A}\}$ denotes the symmetric part of tensor $\mathbf{A}$. It satisfies the equilibrium equation

$$
\operatorname{div} \mathbf{T}=0 .
$$

Complementing the above equations with a constitutive relation for the average dislocation velocity $\mathbf{V}$ as a function of stress and dislocation orientation, and with phenomenological evolution equations for the statistical densities involved in the conventional velocity gradient $\mathbf{L}_{\mathbf{p}}$, one obtains a closed theory in the sense that it contains enough statements to derive uniquely the dynamics of stress and dislocation densities in a bounded domain from boundary and initial conditions. In particular, the direction $\mathbf{d}$ of velocity $\mathbf{V}$ is prescribed as ${ }^{15}$

$$
\begin{gathered}
\mathbf{V}=V \frac{\mathbf{d}}{|\mathbf{d}|}, \quad V \geq 0, \quad \mathbf{d}=\mathbf{b}-\left(\mathbf{b} \cdot \frac{\mathbf{a}}{|\mathbf{a}|}\right) \frac{\mathbf{a}}{|\mathbf{a}|}, \\
\mathbf{b}=\mathbf{X}\left(\mathbf{T}^{\prime} \cdot \boldsymbol{\alpha}\right), \quad \mathbf{a}=\mathbf{X}[\operatorname{tr}(\mathbf{T}) \boldsymbol{\alpha}],
\end{gathered}
$$

to ensure positive dissipation. Here, $\mathbf{T}^{\prime}$ is the stress tensor deviator, $\operatorname{tr}(\mathbf{T})$ is the trace of tensor $\mathbf{T}$, and $\mathbf{X}$ represents the alternating Levi-Civita tensor. Boundary conditions comprise the conventional stress and displacement conditions, as well as the specification of inward fluxes of dislocations. A more detailed account of this overarching framework can be found in Refs. 15-17. When the scale of resolution is commensurate to the (macroscopic) sample size, long-range interactions due to lattice incompatibility may be overlooked. Then tak- 
ing $\boldsymbol{\alpha}=0$ defines a case that can be handled by conventional plasticity. Conversely $\mathbf{L}_{\mathbf{p}}=0$ when all dislocations are resolved. For computational reasons, only small scale systems can then be solved. Taking the time derivative of Eqs. (3)-(5), (11), and (12), and substituting into Eq. (11), one obtains a rate form of these equations

$$
\begin{gathered}
\dot{\mathbf{U}}_{\mathbf{p}}=\boldsymbol{\alpha} \times \mathbf{V}+\mathbf{L}_{\mathbf{p}}, \\
\dot{\boldsymbol{\alpha}}=-\operatorname{curl} \dot{\mathbf{U}}_{\mathbf{p}}, \\
\operatorname{div} \dot{\mathbf{T}}=\operatorname{div} \mathbf{C}_{\mathbf{e}}:\left\{\operatorname{grad} \dot{\mathbf{u}}-\dot{\mathbf{U}}_{\mathbf{p}}\right\}=0 .
\end{gathered}
$$

As the incompatibility Eq. (3) is not explicitly solved in this rate formulation, solutions are limited to initial conditions not involving polar dislocations, but their numerical computation is easier and faster. Conventional plasticity is recovered by taking $\mathbf{V}=0$. Of course the continuity required for $\dot{\mathbf{U}}_{\mathbf{p}}$ in Eq. (14) is only approximately realized in a finite element implementation.

It is perhaps insightful to evoke analogies between dislocation dynamics and eddies dynamics in turbulent flow. ${ }^{22}$ This analogy extends to transport of polar/statistical dislocation densities, as expressed through Eqs. (9) and (10), and large eddy simulations (LES) in the analysis of turbulence. ${ }^{23}$ Turbulent flow is characterized by eddies at all scales. Averaging in space the Navier-Stokes equations provides equations for large resolved eddies, while unresolved ones are dealt with using additional subgrid-scale variables. Closure of the theory is obtained through subgrid phenomenological models featuring scaling character. ${ }^{24}$ In dislocation dynamics, averaging in space secures equations for polar dislocations while providing the link with conventional plasticity: closure for the unresolved variables $\mathbf{L}_{\mathbf{p}}$ derives from wellestablished models for the viscoplasticity of crystalline materials, i.e., relations for forest hardening and lattice rotation having received decades of attention and experimental validation [see below Eqs. (15)-(19)]. Also in contrast to turbulence, scaling behavior is associated with grid scale level, not subgrid scale, as we show below.

Two types of solutions are offered in what follows, in order to provide various insights into the problem. First we conduct a full 3D numerical solution of Eqs. (3) and (8)-(12) in a single crystal by using a Galerkin-least-squares finite element method appropriate for transport problems (see Refs. 15 and 23 for details). These simulations provide for the characterization of intermittency and transport at the specimen scale. At a somewhat smaller scale, we shall also consider a 2D simplified situation with dislocations pertaining to, and gliding in a single slip plane with no out-of-plane motion and projection of the tensile stress into a resolved shear stress. The latter simulations provide for representative behavior of some portion of a slip plane in the single-crystal experiment.

\section{MODEL SIMULATIONS}

The sample geometry and orientation studied by Nittono ${ }^{25}$ serve as a basis for the investigation in the $3 \mathrm{D}$ simulation of
TABLE I. Material parameters used in the 3D $\mathrm{Cu}$ whisker simulation.

\begin{tabular}{cccccc}
\hline \hline$\alpha$ & $b$ & $n$ & $V_{0}$ & $\sigma_{0}$ & \\
\hline 0.35 & $2.5 \times 10^{-10} \mathrm{~m}$ & 20 & $3.5 \times 10^{-8} \mathrm{~m} / \mathrm{s}$ & $3.7 \mathrm{MPa}$ & \\
$C_{0}$ & $C_{1}$ & $C_{2}$ & $C_{11}$ & $C_{12}$ & $C_{44}$ \\
25 & $2.4310^{-5}$ & 3.03 & $170 \mathrm{GPa}$ & $123 \mathrm{GPa}$ & $75.2 \mathrm{GPa}$ \\
\hline \hline
\end{tabular}

the tensile test. The simulation procedure is detailed in Ref. 17 and will be briefly recounted here. A flat $\mathrm{Cu}$ whisker of dimensions $200 \times 30 \times 2400 \mu^{3}$ is clamped to the left end, while the right end is submitted to constant velocity. The applied strain rate is $\dot{\epsilon}_{a}=10^{-3} \mathrm{~s}^{-1}$. The mesh contained 27648 brick elements in a $24 \times 6 \times 192$ arrangement with linear interpolation for the transport equation and quadratic interpolation for the equilibrium equation. The elastic response is taken to be anisotropic with elastic constants $C_{11}$, $C_{12}$, and $C_{44}$. The plastic velocity gradient $\mathbf{L}_{\mathbf{p}}$ follows from the activity of the statistical mobile dislocations on the $12 \mathrm{fcc}$ slip systems

$$
\mathbf{L}_{\mathbf{p}}=\sum_{s} \rho_{m} b V_{s} \mathbf{b}_{\mathbf{s}} \otimes \mathbf{n}_{\mathbf{s}}
$$

where $\rho_{m}$ is the mobile statistical dislocation density, and $\mathbf{b}_{\mathbf{s}}$ and $\mathbf{n}_{\mathbf{s}}$ are the slip system Burgers vector and glide plane normal, respectively. $V_{s}$ is the ensemble dislocation velocity, which follows the power-law relationship

$$
V_{s}=V_{0} \operatorname{sgn}\left(\sigma_{s}\right)\left(\frac{\left|\sigma_{s}\right|}{\sigma_{0}+\sigma_{h}}\right)^{n}, \quad \sigma_{s}=\mathbf{b}_{\mathbf{s}} \otimes \mathbf{n}_{\mathbf{s}}: \mathbf{T} .
$$

Here $\sigma_{s}$ is the resolved shear stress on a glide plane, with reference velocity $V_{0}$, athermal stress $\sigma_{0}$, and stress exponent $n=20$ as material parameters. The threshold stress $\sigma_{h}$ reflects short-range obstacle overcoming. It relates to the statistical forest density $\rho_{f}$ through the usual Taylor relation $\tau_{h}$ $=\alpha \mu b \sqrt{\rho_{f}}$, where $\alpha$ is a nondimensional parameter. Large $n$ values reflect abruptness of dislocation unpinning. The velocity $V$ of polar dislocations is taken as the average of the statistical slip velocity absolute values $\left|V_{s}\right|$ over all slip systems. Hence, the same physics applies to both dislocation species. We adopt evolution equations for $\rho_{m}$ and $\rho_{f}$ as outlined in Ref. 26, with simplifications deemed appropriate for the low strain level achieved in the present simulations,

$$
\begin{gathered}
\dot{\rho}_{m}=\left(C_{1} / b^{2}-C_{2} \rho_{m}\right) \dot{\Gamma}, \\
\dot{\rho}_{f}=\left(C_{0} b|\boldsymbol{\alpha}|+C_{2} \rho_{m}\right) \dot{\Gamma}, \\
\dot{\Gamma}=\left|\dot{\mathbf{U}}_{\mathbf{p}}\right|,
\end{gathered}
$$

where $C_{0}, C_{1}$, and $C_{2}$ are material parameters accounting for the interaction between polar and forest dislocations, the mobile dislocation generation and loss, respectively. $\dot{\Gamma}$ is a scalar measure of plastic slip rate accounting for the combined action of statistical and polar dislocations. The material parameters used in the 3D simulation are listed in Table I. Note 

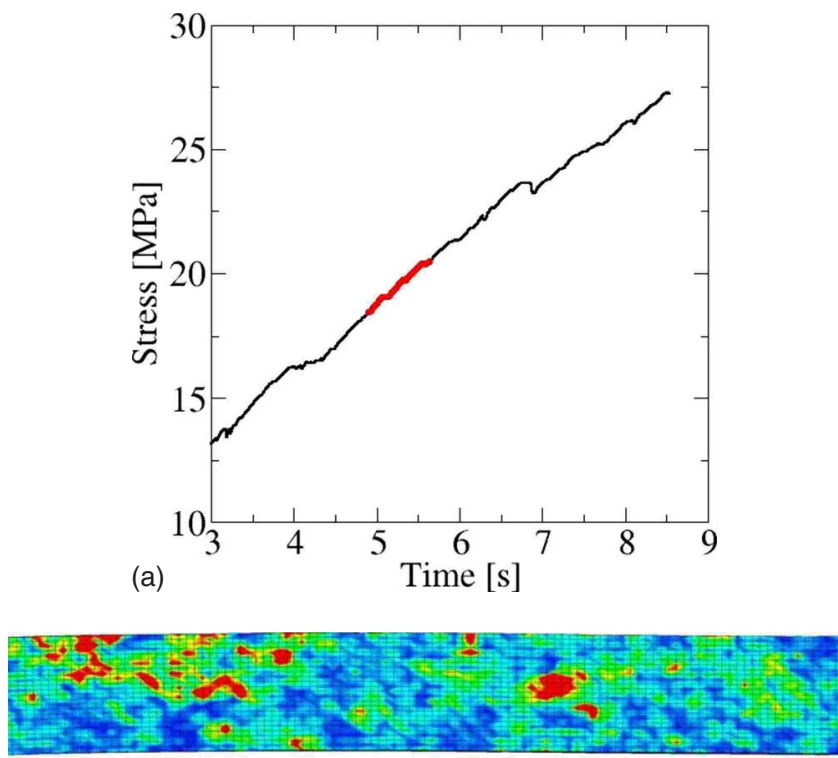

\section{$4.9 \mathrm{~s}$}

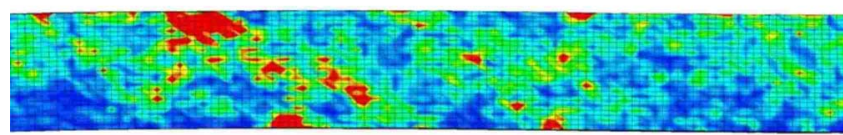

$5.2 \mathrm{~s}$

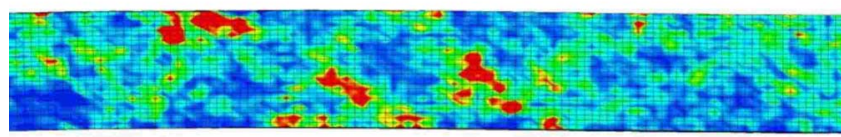

$5.4 \mathrm{~s}$

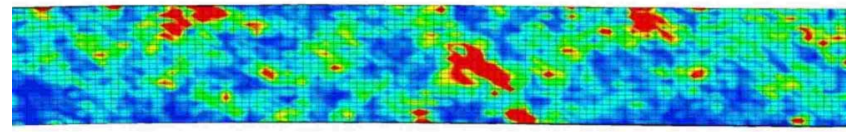

$5.6 \mathrm{~s}$

(b)

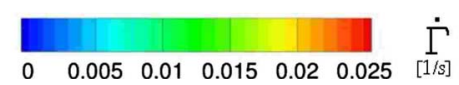

FIG. 3. (Color online) (a) Stress vs time response during stage II linear hardening. The highlighted portion corresponds to the strainrate plots shown in (b). (b) Successive frames of the strain-rate spatiotemporal field along the sample showing intermittent plasticity through dislocation transport.

that there is no inhomogeneity introduced in either material parameters or initial conditions.

The response of the sample is as follows. Elastic loading is followed by a yield drop associated with plastic activity localized near the clamped end, then by a plateau. Thus, inhomogeneity of plastic straining clearly stems from boundary conditions. This prediction of a yield drop is in full agreement with experimental data on $\mathrm{Cu}$ whiskers. ${ }^{25,27,28}$ Along the plateau, the plastic activity spreads over the sample through the motion of a plastic front, before linear homogeneous strain hardening takes place. Details on the propagation of slip during the elastoplastic transition are provided in Ref. 17. In the following, we focus on the intermittency of plastic activity during the eventual linear hardening period, shown in Fig. 3(a). Bursts in stress rate are seen all along the curve. One particular sequence, highlighted in the figure, corresponds to the plots of plastic strain rate shown in

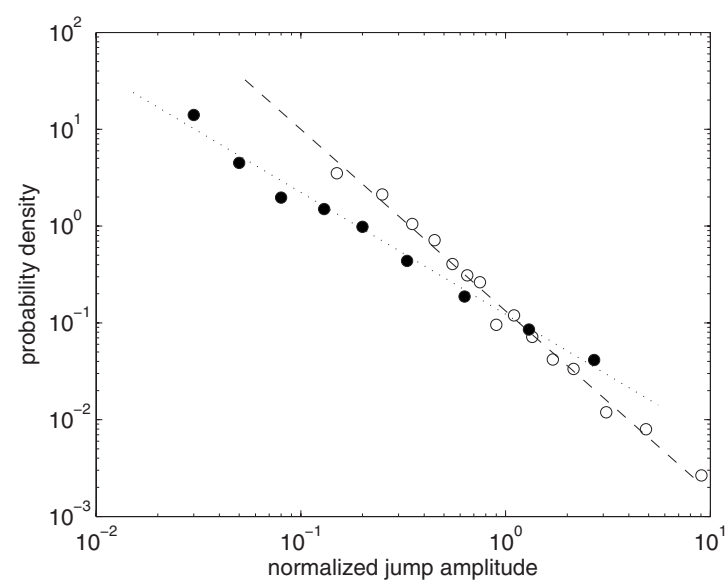

FIG. 4. Probability density of event size. The event size is defined either as a stress drop (filled circles; the dotted trend line shows a $\tau_{s}=1.2$ slope) or the maximum amplitude of stress rate bursts in the linear hardening region (open circles; the dashed trend line shows a $\tau=1.9$ slope).

Fig. 3(b). In this figure, intermittent events are clear with a general progression of plastic activity from left to right of the sample section. Statistical analysis of the bursts occurring in Fig. 3(a) shows power-law scaling with exponent $\tau$ $=1.9 \pm 0.1$ in stress rate burst size distribution and exponent $\tau_{s}=1.2 \pm 0.1$ in stress drop size distribution (see Fig. 4), without inconsistency as shown in Sec. III. However, the small number of stress drops (89) makes it impossible to check accurately for the compatibility of these two exponents. The predicted exponent for stress rate burst size is consistent with our experimental data. Note that the predicted scaling exponent for stress drop size distribution is also consistent with the experimental value $\tau \approx 1.1$ reported in Ref. 29 for $\mathrm{Cu}-10 \% \mathrm{Al}$ single crystals where, however, the addition of solute elements induces the macroscopic plastic instability referred to as the Portevin-Le Chatelier effect, with much larger drop size.

In view of these results, 2D simulations (more tractable than three dimensions) were carried out in order to check for scaling behavior at a smaller scale and for possible invariance of the scaling exponent. In these simulations, a $L_{1}$ $\times L_{2}$ rectangle subjected to constant shear rate $v_{1,3}$ at boundaries (a comma indicates derivation) is considered in the $\left(x_{1}, x_{2}\right)$ glide plane of a $\mathrm{Cu}$ single crystal. The simulation in a $100 \times 100 \mu \mathrm{m}^{2}$ square is referred to as the "reference simulation" in what follows. Elasticity is taken to be isotropic with shear modulus $\mu$. The average velocity $V$ of dislocations in the plane is described with the thermally activated constitutive law

$$
V=V_{0} \exp \frac{-\Delta G_{0}}{k T} \exp \left(\frac{V^{*} \sigma_{s}}{k T\left(1+\sigma_{h} / \sigma_{0}\right)}\right),
$$

where $V_{0}$ is a reference velocity, $\left(\Delta G_{0}, V^{*}, k, T\right)$ is a reference enthalpy, the activation volume, the Boltzmann constant, and the temperature. $\sigma_{s}$ is again the involved shear stress component and $\sigma_{h}$ is the threshold stress for obstacle overcoming. Here Eq. (20) is an alternative to Eq. (16) used 
TABLE II. Initial and boundary conditions, complementary material parameters in $2 \mathrm{D}$ simulations.

\begin{tabular}{cccccc}
\hline \hline$\alpha_{i j}(0)$ & $\rho_{m}(0)$ & $\mu$ & $\sigma_{h}$ & $v_{1,3}$ & $k T / V^{*}$ \\
\hline 0 & $10^{8} \mathrm{~m}^{-2}$ & $40 \mathrm{GPa}$ & $50 \mathrm{MPa}$ & $5 \times 10^{-4} \mathrm{~s}^{-1}$ & $2.27 \mathrm{MPa}$ \\
\hline \hline
\end{tabular}

to describe weak rate sensitivity of the shear stress. In order to mimic the high-resolution extensometry experiments described above, the imposed strain rate is $\dot{\epsilon}_{a}=5 \times 10^{-4} \mathrm{~s}^{-1}$. Since the recording frequency is $10^{3} \mathrm{~Hz}$, the computation time step is set to $10^{-3} \mathrm{~s}$. The mesh size in the finite element simulation is then chosen to respect the Courant condition for numerical stability. ${ }^{23}$ This choice results in a $30 \times 30$ mesh of quadrangular elements. In the initial configuration, polar dislocations are absent and the statistical mobile density is chosen at random about an average value. Since the boundary conditions are homogeneous in this 2D simulation (in contrast to the above 3D simulation), the incompatibility arising from this distribution is initially the only source for polar dislocations. The information on material parameters, initial, and boundary conditions is summed up in Table II.

Figure 5 shows a space-time diagram for strain-rate fluctuations. In qualitative agreement with Fig. 2, spots of intense plastic activity dotted along straight lines are seen. This pattern follows naturally from the development of polar dislocation density by virtue of dislocation transport and internal stress. The velocity obtained from the slopes in Fig. 5 has the order of magnitude observed in experiments (see Fig. 2). At larger strain, the $100 \times 100 \mu \mathrm{m}^{2}$ square shown in Fig. 6(a) displays a heterogeneous structure of loops in the glide plane. The time series obtained for the net shear strain rate at a material point in Fig. 6(a) is plotted in Fig. 6(b). Similarity with the strain-rate signal from extensometry experiments in Fig. 1(b) can be noticed. Figure 6(c) shows the probability density for event size computed from this curve. A scaling distribution is seen, with exponent $\tau \approx 2$ in agreement with the experimental value. This result implies that the fluctuations in Fig. 6(b) are not numerical noise, but reflect instead correlations due to polar dislocation development, longrange stress, and dislocation transport. Refining the mesh size by a factor 2 has no effect on the event size distribution. Similarly, dividing the time step by a factor 10 leads to

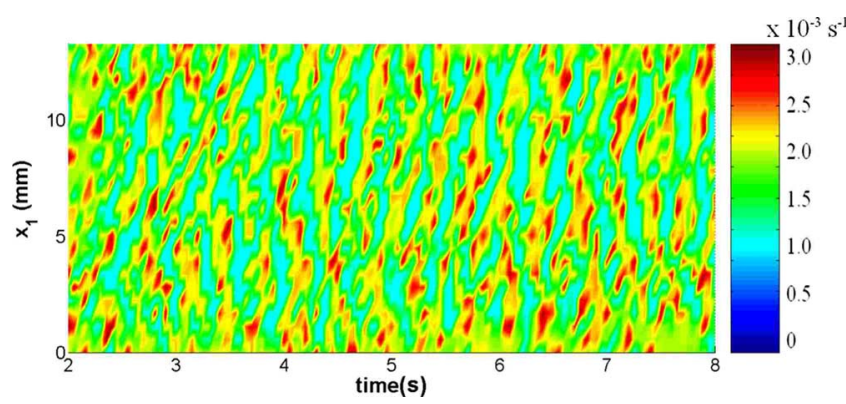

FIG. 5. (Color online) Model predictions for axial $\left(x_{1}\right)$ strainrate fluctuations in a space-time diagram. The sample is a 13 $\times 13 \mathrm{~mm}^{2}$ square in a glide plane subjected to equal shear rates $5 \times 10^{-4} \mathrm{~s}^{-1}$ on both sides. The figure shows the evolution in time of the strain-rate profile seen along the $x_{2}$ direction.
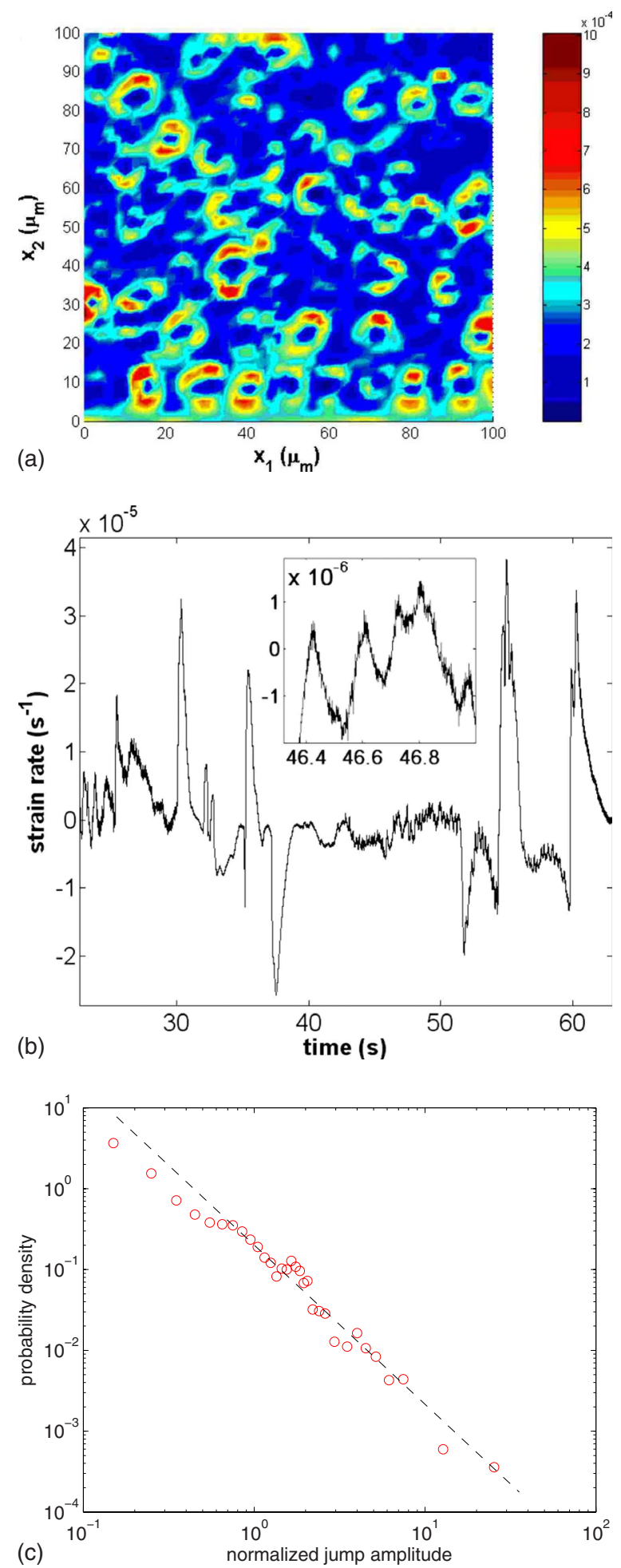

FIG. 6. (Color online) (a) Polar dislocation density distribution showing self-organized loops in a $100 \times 100 \mu \mathrm{m}^{2}$ square in the glide plane at $0.5 \%$ plastic strain. The driving strain rate is 5 $\times 10^{-4} \mathrm{~s}^{-1}$. Note that the maximum fluctuation size is larger than the driving strain rate. (b) Time series (50 000 data points) for the net shear strain rate (i.e., fluctuations about the applied strain rate) at a given point in (a). The closeup in the inset suggests self-similar structure. (c) Probability density of event size at three distinct points in (a). The event size is defined as the maximum strain-rate value during the event. The dotted line shows a $\tau=2$ slope. 
TABLE III. Numerical constants for ice single crystals.

\begin{tabular}{ccccc}
\hline \hline$\mu$ & $b$ & $V_{0}$ & $\sigma_{0}$ & $n$ \\
\hline $3 \mathrm{GPa}$ & $4.5 \times 10^{-10} \mathrm{~m}$ & $3.6 \times 10^{-7} \mathrm{~m} / \mathrm{s}$ & $0.1 \mathrm{MPa}$ & 2 \\
\hline \hline
\end{tabular}

smaller events but their distribution remains unchanged.

We investigated the influence of driving rate, sample size and shape, and mechanical behavior on the statistics of intermittency. To check for driving rate effects, the applied strain rate $\dot{\epsilon}_{a}$ was reduced by a factor 10 , which in turn reduces the nucleation of polar dislocations in the same proportion. Yet, the power law with exponent $\tau=2$ was found for the event size probability density distribution, in agreement with the trend observed in our experiments on $\mathrm{Cu}$ single crystals. Using a slender sample did not have effects either. Possible sample size effects were investigated by uniformly increasing the sample dimensions by a factor 10, while keeping unchanged the applied strain rates. Of course, velocity gradients were reduced by a factor 10 in this process, which leads to lower polar dislocation density and smaller event size. Nevertheless, the exponent $\tau=2$ of the scaling regime was left unchanged. Hence, to the extent that velocity gradients remain large enough to induce polar dislocation development, their variations seem to no avail regarding the statistics of intermittency. Possible influence of material behavior was also investigated by simulating the plasticity of ice single crystals. The geometry and loading conditions of the reference simulation were kept unchanged, but material behavior was switched from thermally activated to viscous drag. Indeed, $\mathrm{Cu}$ and ice have very different crystalline structures and mechanical behavior. Ice is an hcp material with strong anisotropy of slip, which occurs almost exclusively on basal planes..$^{30}$ In single crystals, the average dislocation velocity is ruled by viscous power-law relationship (16) with exponent $n=2$ in stress. ${ }^{31}$ In addition, the typical elastic shear modulus is $\mu=3 \mathrm{GPa}$, much less than the $\mu$ $=40 \mathrm{GPa} \mathrm{Cu}$ value, which has implications on internal stress fields. Parameters $\left(V_{0}, \sigma_{0}\right)$ are identified from experimental data. ${ }^{30,31}$ The threshold stress $\sigma_{h}$ is kept small and constant. Note however that directional (kinematic) hardening arises from dislocation structures building up in the straining process. ${ }^{32}$ All physical parameters for ice single crystals are summarized in Table III. Despite these differences, running the simulation shown in Fig. 6 using ice single-crystal data, still leads to a scaling regime with exponent $\tau=2$ in the event size distribution.

\section{SUMMARY AND CONCLUDING REMARKS}

In this paper, transport of dislocation densities is demonstrated using high-resolution extensometry data gathered in the micrometer to millimeter range from experiments on $\mathrm{Cu}$ single crystals. In particular, propagation along characteristic lines is evidenced in spatiotemporal maps of plastic strain rate, which allows direct measurement of the ensemble dislocation velocity. Intermittency as well as spatiotemporal self-organization of plastic activity is apparent in these maps. Scaling behavior of activity bursts is shown to be robust once the statistical analysis is carefully conducted. It leads to an apparently universal scaling exponent value $\tau \approx 2$ in the event size distribution. Simulations derived from a field dislocation dynamics model dealing with the combined problem of dislocation transport and internal stress field development retrieve intermittency of plastic activity, waviness of its spatiotemporal self-organization, scaling behavior, and universality of the scaling exponent. These features are found at the scale of the sample in the stress vs time response, and at the slip plane level in the strain-rate spatiotemporal field. Intermittency in our model arises from the abruptness of the unpinning transition on short-range obstacles as described through a weakly rate-sensitive stress-velocity relationship. The model implies that both long-range interactions and dislocation transport play a role in the scaling behavior of intermittency. Therefore we present the conjecture that universality of the scaling exponent reflects both aspects. As dislocation transport involves such mechanisms as double cross slip of screw dislocations by-passing short-range obstacles, this conjecture implies that short-range interactions play a significant role in the intermittency of plastic activity. Such a conclusion is fully consistent with observations driven from dislocation dynamics simulations. ${ }^{33}$

\section{ACKNOWLEDGMENTS}

A.J.B. received support under U.S. Department of Energy under Grant No. DEFG03-02-NA00072 and the Center for Simulation of Advanced Rockets at the University of Illinois at Urbana-Champaign (UIUC), U.S. DOE under Subcontract No. B341494. A.J.B., C.F., and V.T. benefited from exchanges under a joint agreement between Centre National de la Recherche Scientifique and UIUC. We thank Yuri Chumlyakov for providing a $\mathrm{Cu}$ crystal and Russell J. McDonald for his help in the experiments.
${ }^{1}$ E. Kröner, Erg. Angew. Math 5, 1 (1958).

${ }^{2}$ T. Mura, Philos. Mag. 8, 843 (1963).

${ }^{3}$ A. Acharya, J. Mech. Phys. Solids 49, 761 (2001).

${ }^{4}$ L. B. Zuev, Ann. Phys. 16, 286 (2007).

${ }^{5}$ R. Becker and E. Orowan, Z. Phys. 79, 566 (1932).

${ }^{6}$ J. Weiss and J. R. Grasso, J. Phys. Chem. 101, 6113 (1997).

${ }^{7}$ M. C. Miguel, A. Vespignani, S. Zapperi, J. Weiss, and J. R.
Grasso, Nature (London) 410, 667 (2001).

${ }^{8}$ D. M. Dimiduk, C. Woodward, R. LeSar, and M. D. Uchic, Science 312, 1188 (2006).

${ }^{9}$ S. Brinckmann, J. Y. Kim, and J. R. Greer, Phys. Rev. Lett. 100, 155502 (2008).

${ }^{10}$ J. Weiss, T. Richeton, F. Louchet, F. Chmelik, P. Dobron, D. Entemeyer, M. Lebyodkin, T. Lebedkina, C. Fressengeas, and R. 
J. McDonald, Phys. Rev. B 76, 224110 (2007).

${ }^{11}$ F. F. Csikor, C. Motz, D. Weygand, M. Zaiser, and S. Zapperi, Science 318, 251 (2007).

${ }^{12}$ M. Koslowski, R. LeSar, and R. Thomson, Phys. Rev. Lett. 93, 125502 (2004).

${ }^{13}$ M. Zaiser, Adv. Phys. 55, 185 (2006).

${ }^{14}$ J. P. Sethna, K. Dahmen, and C. R. Myers, Nature (London) 410, 242 (2001).

${ }^{15}$ A. Acharya and A. Roy, J. Mech. Phys. Solids 54, 1687 (2006).

${ }^{16}$ V. Taupin, S. Varadhan, C. Fressengeas, and A. J. Beaudoin, Acta Mater. 56, 3002 (2008).

${ }^{17}$ A. Acharya, A. J. Beaudoin, and R. Miller, Math. Mech. Solids 13, 292 (2008).

${ }^{18}$ E. M. Nadgorny, Dislocation Dynamics and Mechanical Properties of Crystals (Pergamon, Oxford, 1988).

${ }^{19}$ R. A. White and K. A. Dahmen, Phys. Rev. Lett. 91, 085702 (2003).

${ }^{20}$ F. Omori, J. Coll. Sci., Imp. Univ. Tokyo 7, 111 (1894).

${ }^{21}$ J. F. Nye, Acta Metall. 1, 153 (1953).
${ }^{22}$ M. J. Marcinkowski, Phys. Status Solidi B 152, 9 (1989).

${ }^{23}$ S. Varadhan, A. J. Beaudoin, A. Acharya, and C. Fressengeas, Modell. Simul. Mater. Sci. Eng. 14, 1245 (2006).

${ }^{24}$ C. Meneveau and J. O’Neil, Phys. Rev. E 49, 2866 (1994).

${ }^{25}$ O. Nittono, Jpn. J. Appl. Phys. 10, 188 (1971).

${ }^{26} \mathrm{~S}$. Varadhan, A. J. Beaudoin and C. Fressengeas, Proceeding of Science, SMPRI2005, 2006, p. 004.

${ }^{27}$ S. S. Brenner, J. Appl. Phys. 28, 1023 (1957).

${ }^{28}$ Y. Gotoh, Phys. Status Solidi A 24, 305 (1974).

${ }^{29}$ G. Ananthakrishna, S. J. Noronha, C. Fressengeas, and L. P. Kubin, Phys. Rev. E 60, 5455 (1999).

${ }^{30}$ P. Duval, M. F. Ashby, and I. Anderman, J. Phys. Chem. 87, 4066 (1983).

${ }^{31}$ C. Shearwood and R. W. Whitworth,Philos. Mag. A 64, 289 (1991).

${ }^{32}$ V. Taupin, S. Varadhan, J. Chevy, C. Fressengeas, A. J. Beaudoin, M. Montagnat, and P. Duval, Phys. Rev. Lett. 99, 155507 (2007).

${ }^{33}$ B. Devincre, T. Hoc, and L. P. Kubin, Science 320, 1745 (2008). 